\title{
Dynamical electroweak symmetry breaking by quasiconformal technicolour theories
}

\section{Dennis D. Dietrich}

Centre for Particle Physics Phenomenology (CP3-Origins), University of Southern Denmark,

Odense, Denmark

\begin{abstract}
In technicolour (TC) theories, the electroweak (EW) symmetry is broken by chiral symmetry breaking $(\chi \mathrm{SB})$ in an additional strongly interacting sector, added to the standard model (SM) without elementary Higgs sector. Quasiconformal, that is, walking technicolour (WTC) models with matter in higher representations of the technicolour gauge group are viable candidates for breaking the electroweak symmetry dynamically. They are not at odds with available electroweak precision data. Here, we start with a brief introduction into dynamical electroweak symmetry breaking by technicolour theories. Subsequently, we discuss the phase diagram of strongly interacting theories in the $N_{c}-N_{f}$-plane or, equivalently, the $d_{R}$ - $N_{f}$-plane, where $N_{c}$ stands for the number of colours, $N_{f}$ for the number of flavours, and $d_{R}$ for the dimension of the representation; and how to relate said phase diagram to the task of finding candidates for quasiconformal technicolour models. Continuing from there, we select the prime candidates [among them Minimal Walking Technicolour (MWT)] by using constraints from available electroweak precision data like, for example, bounds on flavour changing neutral currents (FCNCs), oblique parameters and the masses $m_{\pi}$ of extra Nambu-Goldstone modes. The latter issue is also linked to the stability of the vacuum. We discuss the features of selected candidates in more detail. Their signals can be detected at the LHC, they feature dark matter (DM) candidates, and they are being studied actively with lattice computations, nonperturbative $\beta$-function techniques as well as AdS/CFT methods.
\end{abstract}

The 2009 Europhysics Conference on High Energy Physics,

July 16 - 222009

Krakow, Poland 

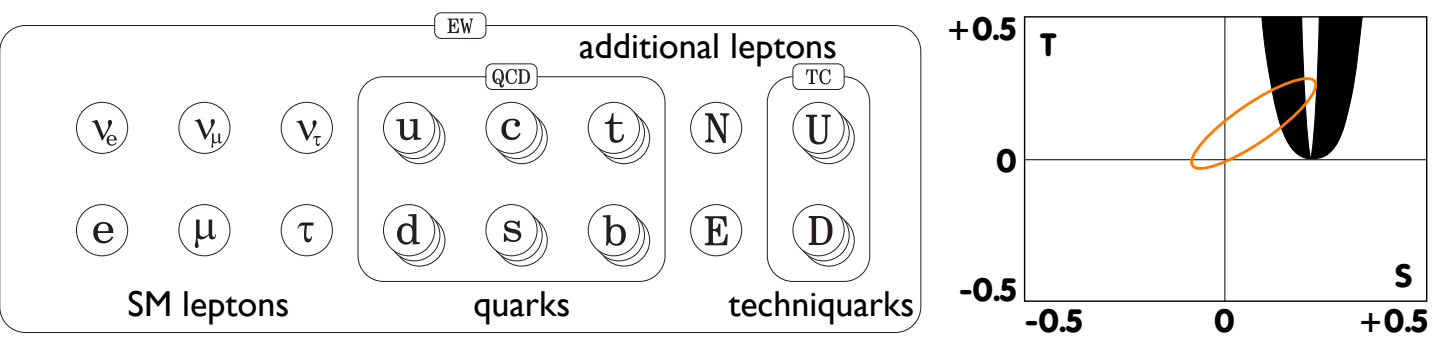

Figure 1: Lhs: TC using the example of MWT. $\underline{\text { Rhs: }}$ MWT vs. oblique parameters [1, 2].

In the SM the Higgs breaks the EW symmetry while preserving gauge invariance, perturbative renormalisability and unitarity. It is, however, as yet undiscovered and would be the first elementary scalar. On all other occasions in which we observe a Higgs phenomenon, like in superconductivity, the scalar dof is composite. There is no dynamical explanation for its negative mass term. As elementary scalar its mass is not symmetry protected and is thus quadratically divergent.

A way to break the EW symmetry (dynamically) without any elementary scalars is TC [3]. In TC, the EW symmetry is broken through $\chi \mathrm{SB}$ in a strongly interacting, EWly charged sector with $N_{f}$ technifermions added to the SM without its Higgs sector. The gauge group is $\mathscr{G}_{\mathrm{SM}} \times \mathscr{G}_{\mathrm{TC}}$. [See Fig. 1 (lhs).] For the corresponding pion decay constant we have $N_{f} f_{\pi}^{2}=2 \Lambda_{\mathrm{ew}}^{2}$, where $\Lambda_{\mathrm{ew}}$ is the EW scale. 3 of the possibly more pions are absorbed as longitudinal dofs of the weak gauge bosons. The mass of the composite scalar is protected by the chiral symmetry $(\chi \mathrm{S})$. At variance with the SM, the unitarity of the theory is not the sole responsibility of this composite scalar.

Available EW precision data requires that the TC sector have walking dynamics to reconcile the creation of the mass of the top quark through extended TC [3] interactions with the bounds on FCNCs. A small matter content is demanded by the bounds on the oblique parameters [4]. Potential additional pions must be sufficiently massive to escape the direct detection limits. The alignment of the EW gauge group in the flavour symmetry group must be stable [5].

A walking theory is one whose matter content is such that the $\chi \mathrm{S}$ breaks just before an IR fixed point is reached. In that case, just before the $\chi \mathrm{S}$ breaks, the $\beta$-function is almost zero. [See case E in Fig. 2 (lhs).] Consequently, the coupling constant stays constant for a big range of scales, which enhances the techniquark condensate through renormalisation effects. For constructing such a theory we can vary the gauge group $\left(N_{c}\right), N_{f}$, and the rep of the gauge group wrt which these flavours transform. For $S U\left(N_{c}\right)$ gauge groups and without partial gauging [6], we find [7] the lower bounds of the conformal windows in Fig. 2 (rhs). Walking theories lie slightly below these lines.

For walking theories, the oblique $S$-parameter is reduced wrt its perturbative value $12 \pi S_{\text {pert }}=$ $d_{R} N_{f}^{g}$. Experimental data requires it to be small, hence, using $S_{\text {pert }}$ is conservative. Accordingly, the most favourable model is MWT [6,8] with 2 adjoint flavours of $S U(2)$ plus 2 leptons to avoid a Witten anomaly. For Fig. 1 (rhs), we vary their masses between 1 and $10 m_{Z}$, compute the oblique $S$ and $T$ parameters, and find considerable overlap with the experimental 68\%-confidence ellipse.

The adjoint is a real rep and the flavour symmetry of MWT is enhanced to $S U(4) \rightarrow S O(4)$ leading to 9 pions. The 3 technimesons are absorbed as longitudinal dofs of the weak gauge bosons and the remaining 6 are technibaryons. Depending on the hypercharge assignment (constrained but not fully fixed by requiring the absence of gauge anomalies), some of the latter can be electrically 

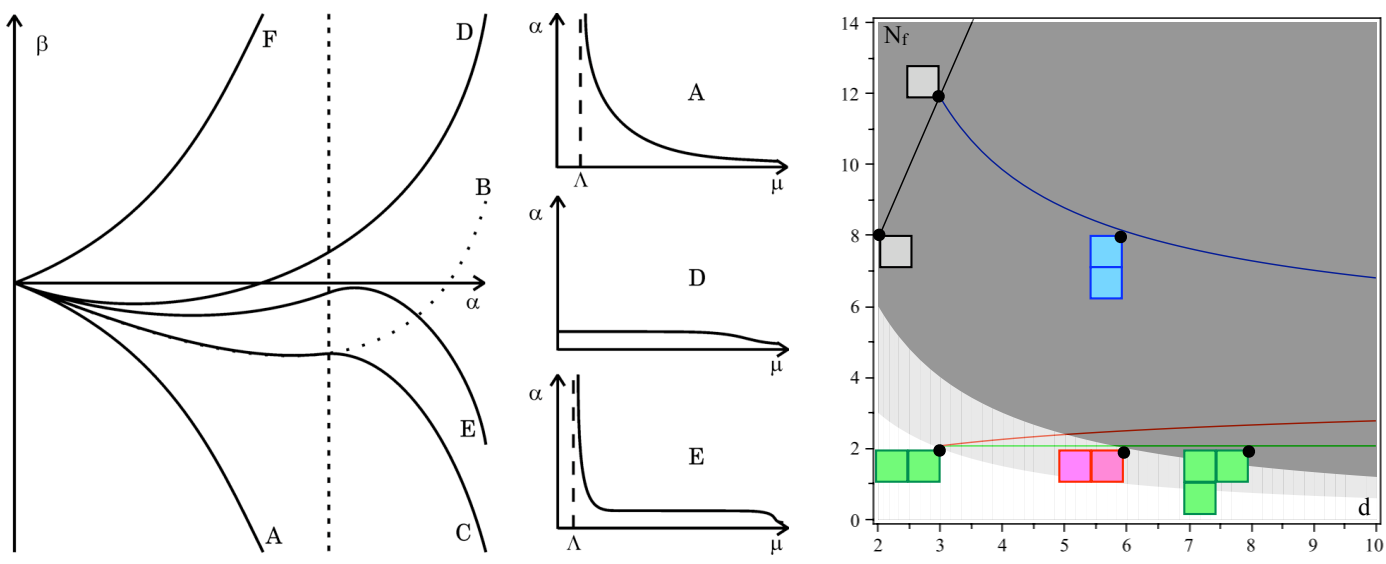

Figure 2: Lhs: Behaviour of the $\beta$-function as a function of the coupling $\alpha$ and of the coupling as a function of the energy scale $\mu$, depending on the matter content of the theory, from [9]. A) Little matter; B) perturbative Caswell-Banks-Zaks fixed point; C) actual shape due to $\chi \mathrm{SB}$; D) realised fixed point; E) walking case; F) loss of asymptotic freedom. Short-dashed line: critical value of the coupling for $\chi \mathrm{SB}$. Rhs: Lower bounds of the conformal window in the $d_{R}-N_{f}$-plane for the (from top to bottom) fundamental (black), 2-index-antisymmetric (blue), 2-index-symmetric (red) or adjoint (green) rep of $S U\left(N_{c}\right)$. White area: $\pi S_{\text {pert }}<1 / 2$, light-gray: $\pi S_{\text {pert }}<1$. The Young tableaux indicate some walking theories [7].

neutral and thus, candidates for DM [10]. The EW contribution to $m_{\pi}$ is typically $>m_{Z}[11]$, which takes them outside the direct exclusion limit for technimesons. They are, however, technibaryons, which can only be produced in pairs. The positive contribution to $m_{\pi}^{2}$ stabilises the embedding of the EW gauge group in the flavour symmetry group, which ensures the correct breaking of the former. The 2 flavours of next-to-MWT transform under the [non(pseudo)real] 2-index symmetric rep of $S U(3)$. The corresponding basic $S U(2)_{L} \times S U(2)_{R} \rightarrow S U(2)_{V}$ breaking yields only the 3 longitudinal modes of the weak gauge bosons and the correct vacuum alignment is no issue.

Dynamical electroweak symmetry breaking by WTC is feasible. Bounds on FCNCs, oblique parameters, and $m_{\pi}$ can be reconciled with the generation of SM fermion masses by walking together with techniquarks in higher reps of the TC gauge group. WTC models can be detected at the LHC [12] and feature DM candidates [10]. WTC theories are actively investigated with nonperturbative $\beta$-function techniques [9, 14], AdS/CFT methods [13] and lattice computations [15]. There are many more candidates beyond MWT \& NMWT [7] (with full or partial gauging [6]).

The work of DDD was supported by the Danish Natural Science Research Council.

\section{References}

[1] D. D. Dietrich, Composite Higgs from higher reps in E. Accomando et al., Workshop on CP Studies and Non-standard Higgs Physics, CERN-2006-009, arXiv:hep-ph/0608079.

[2] ALEPH, DELPHI, L3, OPAL, SLD Collaborations, the LEP EW Working Group, the SLD EW, heavy flavour groups, Precision EW measurements on the Z resonance, Phys. Rept. 427 (2006) 257.

[3] Reviews: K. Lane, Two lectures on TC, arXiv:hep-ph/0202255; C. T. Hill and E. H. Simmons, Strong dynamics and EW symmetry breaking, Phys. Rept. 381 (2003) 235 [Erratum-ibid. 390 (2004) 553]; F. Sannino, Dynamical Stabilization of the Fermi Scale: Phase Diagram of Strongly Coupled Theories for (Minimal) WTC and Unparticles, arXiv:0804.0182 [hep-ph]. 
[4] M. Peskin and T. Takeuchi, An EW constraint on a strongly interacting Higgs sector, PRL 65 (1990) 964.

[5] M. Peskin, The alignment of the vacuum in theories of TC, NPB 175 (1980) 197.

[6] D. D. Dietrich, F. Sannino and K. Tuominen, Light composite Higgs from higher reps versus EW precision measurements: Predictions for LHC, PRD 72 (2005) 055001.

[7] D. D. Dietrich and F. Sannino, Conformal window of $S U(N)$ gauge theories with fermions in higher dimensional reps, PRD 75 (2007) 085018.

[8] F. Sannino and K. Tuominen, Techniorientifold, PRD 71 (2005) 051901; D. D. Dietrich, F. Sannino and $\mathrm{K}$. Tuominen, Light composite Higgs and precision $E W$ measurements on the $Z$ resonance: An update, PRD 73 (2006) 037701.

[9] D. D. Dietrich, A massive $\beta$-function, arXiv:0908.1364 [hep-th].

[10] S. B. Gudnason, C. Kouvaris and F. Sannino, DM from new TC Theories, PRD 74 (2006) 095008; C. Kouvaris, Dark Majorana Particles from the Minimal WTC, PRD 76 (2007) 015011; WIMP Annihilation and Cooling of Neutron Stars, PRD 77 (2008) 023006; The Dark Side of Strong Coupled Theories, PRD 78 (2008) 075024; M. Y. Khlopov and C. Kouvaris, Strong Interactive Massive Particles from a Strong Coupled Theory, PRD 77 (2008) 065002; Composite DM from a model with composite Higgs boson, PRD 78 (2008) 065040; K. Belotsky, M. Khlopov and C. Kouvaris, Muon Flux Limits for Majorana DM Particles, PRD 79 (2009) 83520; T. A. Ryttov and F. Sannino, Ultra Minimal TC and its DM TIMP, PRD 78 (2008) 115010; R. Foadi, M. T. Frandsen and F. Sannino, TC $D M$, arXiv:0812.3406 [hep-ph]; E. Nardi, F. Sannino and A. Strumia, Decaying DM can explain the $e^{+} e^{-}$excesses, JCAP 0901 (2009) 043.

[11] D. D. Dietrich and M. Järvinen, $m_{\pi}$ in quasiconformal gauge field theories, PRD 79 (2009) 057903.

[12] A. Belyaev, R. Foadi, M. T. Frandsen, M. Järvinen, F. Sannino and A. Pukhov, TC Walks at the LHC, PRD 79 (2009) 035006.

[13] E.g., D. D. Dietrich and C. Kouvaris, Constraining vectors and axial-vectors in WTC by a holographic principle, PRD 78 (2008) 055005; Generalised bottom-up holography and WTC, PRD 79 (2009) 075004; D. D. Dietrich, M. Järvinen and C. Kouvaris, Linear confinement without dilaton in bottom-up holography for WTC, arXiv:0908.4357 [hep-ph].

[14] T. A. Ryttov and F. Sannino, Supersymmetry Inspired QCD Beta Function, PRD 78 (2008) 065001; Conformal House, arXiv:0906.0307 [hep-ph]; F. Sannino, Conformal Windows of $\operatorname{SP}(2 N)$ and $S O(N)$ Gauge Theories, PRD 79 (2009) 096007; QCD Dual, arXiv:0907.1364 [hep-th].

[15] E.g., S. Catterall and F. Sannino, Minimal walking on the lattice, PRD 76 (2007) 034504; S. Catterall, J. Giedt, F. Sannino and J. Schneible, Phase diagram of SU(2) with 2 flavors of dynamical adjoint quarks, JHEP 0811 (2008) 009; L. Del Debbio, M. T. Frandsen, H. Panagopoulos and F. Sannino, Higher reps on the lattice: perturbative studies, JHEP 0806 (2008) 007; L. Del Debbio, A. Patella and C. Pica, Higher reps on the lattice: numerical simulations. SU(2) with adjoint fermions, arXiv:0805.2058 [hep-lat]; Y. Shamir, B. Svetitsky and T. DeGrand, Phys. Rev. D 78 (2008) 031502; L. Del Debbio, B. Lucini, A. Patella, C. Pica and A. Rago, Conformal vs confining scenario in SU(2) with adjoint fermions, arXiv:0907.3896 [hep-lat]; Z. Fodor, K. Holland, J. Kuti, D. Nogradi and C. Schroeder, Nearly conformal gauge theories in finite volume, arXiv:0907.4562 [hep-lat]. 